\title{
Modern Power System Dynamics, Stability and Control
}

\author{
Antonio T. Alexandridis(1) \\ Department of Electrical and Computer Engineering, University of Patras, 26504 Patras, Greece; \\ a.t.alexandridis@ece.upatras.gr
}

Received: 13 July 2020; Accepted: 20 July 2020; Published: 24 July 2020

\begin{abstract}
This Special Issue of Energies, "Modern Power System Dynamics, Stability and Control", addresses the core problem of deploying novel aspects in the analysis of modern power systems as these are composed after the high penetration of distributed generation (DG) with different renewable energy sources (RES). The focus is given either on the new whole power and control system configuration or on individual cases of DG sources, power converters and other general or specific plants and devices. The problem can be tackled with different methodologies and may have several, more or less valuable and complicated solutions. The twenty-three accepted papers certainly offer a good contribution in a wide range of applications; they are extended from basic system theory perspectives, fundamental nonlinear analysis tools and novel modeling deployments to some interesting particular system and control issues.
\end{abstract}

\section{Introduction}

Power systems are continuously changing to effectively incorporate distributed generation (DG) with high levels of penetration of different renewable energy sources (RES), such as wind turbine or photovoltaic (PV) systems. The transition from the conventional electricity grid towards the "smart grid" has introduced continuous advancements in the electrical network infrastructure and the deployment of new emerging monitoring, communication, computation, and control technologies. In order to enhance grid stability, reliability, resilience, and efficiency, all these system and management innovations should be effectively coordinate. Power electronic converters play a key role in the system, operating as controlled power interface devices and allowing local control of both directions of power flow, typically at notable distances from the main grid. RES installations, as well as energy storage systems, are connected to power electronic devices either individually or as a part of new structures such as micro-grids. In the new structure of modern power systems, the RES dynamics and control, the DG and micro-grid operation and stability in islanding or grid-connected mode, are of great importance, whereas HVDC links are vital for balancing the power of the grid. As the rotating system inertia is reduced, frequency response issues become essential and the intermittent nature of RES mandates new ways of regulating voltage. To confront the many challenges, new modeling aspects that take into account the fast and slow RES dynamics and the control capabilities of the power electronic interfaces are needed. Emerging issues involve advanced decentralized control schemes capable of regulating large numbers of distributed sources and loads without adverse impacts. Stability and convergence to the desired equilibrium constitutes another significant point that requires new reliable and efficient for large scale systems, methods of analysis, based on linear and, preferably, nonlinear systems theory. In this frame, the main topics of interest of this Special Issue include:

- Power electronic converters as controlled power interface devices;

- Dynamics and stability of power controlled RES (wind turbines, PV systems, etc.);

- Improvements in power system stability under high levels of penetration of RES;

- HVDC interconnections: modeling and control; 
- DG dynamics and control, integrated with RES and energy storage devices;

- Microgrids (ac or dc) in stand-alone or grid-connected mode;

- Novel aspects of model deployment and nonlinear stability analysis of modern power systems;

- Innovative PI and/or P cascaded controllers for use in DG and RES installations;

- Stability of PLL driven controllers;

- Frequency and voltage droop-based control;

- Ancillary services.

\section{Published Papers Highlights}

This Editorial article provides a summary of the Special Issue of Energies, covering the published papers [1-23] which address several of the topics mentioned in the Introduction. Table 1 identifies the most relevant topics in each published paper. 
Table 1. Topics covered in each publication.

\begin{tabular}{|c|c|c|c|c|c|c|c|c|c|c|c|c|c|c|c|c|c|c|c|c|c|c|c|}
\hline \multirow[t]{2}{*}{ Topic } & \multicolumn{23}{|c|}{ Publication } \\
\hline & [1] & [2] & [3] & [4] & [5] & [6] & [7] & [8] & [9] & [10] & [11] & [12] & [13] & [14] & [15] & [16] & [17] & [18] & [19] & [20] & [21] & [22] & [23] \\
\hline Converters & & $x$ & & & $x$ & $x$ & & $x$ & $x$ & & & $x$ & & $x$ & $x$ & & $x$ & & & & & & $x$ \\
\hline RES & $x$ & $x$ & & $x$ & $x$ & & & $x$ & $x$ & & & & $x$ & & $x$ & & & & & & & & $x$ \\
\hline DG & $x$ & $x$ & & $x$ & & & & & & $x$ & $x$ & & $x$ & $x$ & $x$ & & $x$ & & & & $x$ & & $x$ \\
\hline Microgrids & $x$ & & & & & & & & & & & & & $x$ & & & & & & & & & \\
\hline HVDC & & & & & & & & & & & $x$ & $x$ & & & & & & & & & & & \\
\hline Modeling & $x$ & $x$ & $x$ & & $x$ & $x$ & $x$ & & & $x$ & $x$ & $x$ & & $x$ & & $x$ & $x$ & $x$ & $\mathrm{x}$ & $x$ & $x$ & $x$ & $x$ \\
\hline Control & & $x$ & $x$ & & $x$ & & $x$ & $x$ & & & & $x$ & & $x$ & & $x$ & & $x$ & $\mathrm{x}$ & & & $\mathrm{x}$ & \\
\hline Stability & $x$ & $x$ & $x$ & & & & & & $x$ & & & & & $x$ & & $x$ & & $x$ & & & & & \\
\hline Total & 5 & 6 & 3 & 2 & 4 & 2 & 2 & 3 & 3 & 2 & 3 & 4 & 2 & 6 & 3 & 3 & 3 & 3 & 2 & 1 & 2 & 2 & 4 \\
\hline
\end{tabular}


As shown in Table 1, most of the publications focus on modeling while topics on control methodologies and DG applications follow. Six of the papers involve more than four of the Special Issue main topics.

The twenty-three articles have been selected after a peer review process and we are thankful to all the eighty-six authors from several countries (in alphabetic order: Abu Dhabi, Australia, China, Denmark, Finland, Germany, Greece, The Netherlands, United Kingdom, USA, Vietnam) for their contribution to the Special Issue.

In the work presented in [1], Konstantopoulos et al. propose a novel framework for analyzing and expanding research and innovation results that are essential in managing, controlling and operating complex, large scale, power systems under a holistic insight. Modern power systems are deployed in the frame of a cyber-physical system (CPS) architecture that clearly describes how a dedicated cyber layer is deployed to manage and interact with comprehensive multiple physical layers. In particular, the measurement, communication, computation, control mechanisms, and tools installed at different hierarchical frames that are required to consider and modulate the social/environmental necessities, as well as the electricity market management, the regulation of the electric grid, and the power injection/absorption of the controlled main devices and distributed energy resources, are all incorporated in a common CPS framework. Finally, a CPS-based example of a real-world industrial micro-grid is presented.

A novel generalized dynamic representation and full scale modeling of a modern power system is proposed by Papageorgiou et al. in [2]. The developed formulation results in a holistic nonlinear dynamic description, defined here as controlled impedance-admittance-torque (CIAT) model that is extended to include in detail: the well-known impedance-admittance network model, the power converter devices by considering the controlled power electronic dynamics and the electrical machines by inserting their full electromechanical dynamics. The model is deployed in state space and features common structural characteristics that enable the compression of the accurate analytic power system dynamic description into a matrix-based generic nonlinear model in a form easily used for nonlinear studies of such large-scale systems which are presented in [3]. A modern power system example with different DERs is analyzed by this method and is extensively simulated to verify the validity of the proposed method under varying operational and topology conditions.

Contribution [3], proposed by Alexandridis, constitutes the new theoretical background for nonlinear stability studies of a system enforced by external known or unknown inputs. The aim is twofold: Firstly, to substantially complete the theoretical infrastructure by establishing globally valid sufficient conditions for externally enforced nonlinear systems that converge to nonzero equilibriums and, secondly, to deploy an efficient method easily applicable on practical problems as it is analyzed in detail on a typical power system example. A rigorous nonlinear analysis is developed that decisively proves the sufficiency of input-to-state stability (ISS) notion for the state of a system to converge at nonzero equilibrium.

From a stability point of view, Liang Chen et al. [4] provide insights into the deployment of an aggregated small-signal impedance model of wind farms suitable for the analysis between them and weak grids with high line impedance. The impacts of the reference frame mismatch between a local small-signal impedance model and a global one on the accuracy of aggregated impedance and the accuracy of impedance-based stability analysis are considered. The results reveal that the impact is related to the power distribution of the studied network and the influence of mismatch on stability analysis becomes subtle when subsystems are balanced loaded. This is a common configuration in many practical applications.

Xunjun Chen et al. [5] presented a harmonic stability analysis of doubly-fed induction generators (DFIGs) wherein it is taken into account the double pulse width modulation (PWM) converter, one of the main harmonic sources in DFIGs. Aiming at dual PWM converters, which include the grid side converter (GSC) and the rotor side converter (RSC), this paper divides converters into two parts: circuit modules and control modules. Closed-loop input impedance models of each module are then derived 
by means of transfer functions with the stability of the system to be readily predicted through Nyquist diagrams. The contribution of parameters to the system's harmonic stability are also identified.

The influence of grid impedance which is an important parameter affecting the control performance of grid-connected power converters is addressed in [6] by Luhtala et al. This paper points out challenges and errors in grid impedance identification, caused by parallel converters and their internal control functions, such as grid-voltage support. Experimental grid-impedance measurements are shown from the power hardware-in-the-loop setup developed at DNV-GL Flexible Power Grid Lab.

With a focus on the need for simple, accurate performance models of wind turbine generators (WTGs), photovoltaic (PV) plants, and battery energy storage systems (BESS) for various hybrid power plant (HPP) studies, Petersen et al. in [7] propose reduced-order models with HPP controls that minimize the complexity and computation effort of simulation platforms. The models are validated through field measurements and are effective to target power system integration studies involving active and reactive power, as well as frequency and voltage regulation. Field measurements of two Vestas WTGs, one 1-MW PV plant, and one 1-MW/1-MWh BESS are used for model validation.

Jauch et al. [8] present findings of a study on continuous feed-in management and continuous synthetic inertia contribution with wind turbines. A realistic case study, based on real measurements, is outlined. The study shows that continuous feed-in management can be combined well with continuous inertia provision. There are hardly any negative consequences for the wind turbine. The benefits for the grid are convincing, both in terms of increased system utilization and in terms of provided inertia. It is concluded that wind turbines can eventually be used to enhance angular stability in a power system.

In [9], Bo Wang et al. focus on the dynamic analysis of a grid-tied voltage source converter (GVSC) during electromechanical oscillations. A small-signal model and an input-output transfer function matrix are constructed to reveal the passive response of the GVSC on the basis of the power equation in the $d-q$ coordinate system. The frequency response in the electromechanical bandwidth is described to reflect the dynamic behavior of the GVSC. The effects of the operation parameters, i.e., the grid strength, reference value of the control system, and grid voltage, on the dynamic behavior of the GVSC in the electromechanical bandwidth, are investigated using frequency domain sensitivity. The IEEE 10-machine power system simulation is performed, and the power hardware-in-the-loop platform with the GVSC is applied to validate the analysis.

Peng et al. in [10] propose a new probabilistic power flow method for the hybrid AC/VSC-MTDC (Voltage Source Control-Multiple Terminal Direct Current) grids, which is based on the combination of ninth-order polynomial normal transformation (NPNT) and inherited Latin hypercube sampling (ILHS) techniques. NPNT is utilized to directly handle historical records of uncertain sources to build the accurate probability model of random inputs and ILHS is adopted to propagate the randomness from inputs to target outputs. Regardless of whether the underlying probability distribution is known or unknown, the proposed method has the ability to adaptively evaluate the sample size according to a specific operational scenario of the power system. The modified IEEE 118-bus system is used to demonstrate the effectiveness and superiority of the proposed method.

Furthermore, in [11], Xianqi Li, presents a probabilistic small signal stability assessment methodology to select the best locations for multi-infeed high-voltage direct current (HVDC) systems in AC grids. The Latin hypercube sampling-based Monte Carlo simulation approach is taken to generate the stochastic operation scenarios of power systems with the consideration of several stochastic factors, i.e., load demand and power generation. The damping ratio of the critical oscillation modes and the controllability of power injection to oscillation modes are analyzed by the probabilistic small signal stability and a probabilistic index is proposed.

Ying Wang et al. in [12] focus on effects of commonly proposed control modes for a voltage source converter (VSC)-based HVDC systems. The influence of VSC-HVDC with different reactive power control strategies on transient stability and dynamic stability of the AC system is studied. It is shown that compared to constant reactive power control, AC voltage margin control can provide 
voltage support to enhance the transient angle stability. However, the fluctuant reactive power injected into a weak AC system may adversely affect power system oscillation damping for VSC-HVDC with AC voltage margin control, if the parameters of the controller are not optimized to suppress the low-frequency oscillation.

An innovative framework termed phasor-based control (PBC) is proposed in [13] by von Meier et al., to facilitate the integration of heterogeneous and intermittent distributed energy resources (DER) on the electric grid. PBC presents a unified approach that is agnostic to optimization criteria and to the particular characteristics of participating resources. High-precision voltage phasor measurements and initial results suggest that $\mathrm{PBC}$ has significant potential to support stable and resilient grid operations in the presence of arbitrarily high penetrations of DER.

In [14], Paspatis et al. propose and analyze a novel nonlinear current-limiting controller for three-phase grid-tied droop-controlled inverters that is capable of offering voltage support during balanced and unbalanced grid voltage drops. The proposed controller introduces a unified structure under both normal and abnormal grid conditions operating as a droop controller or following the recent fault-ride-through requirement to provide voltage support. Asymptotic stability for any equilibrium point of the closed-loop system in the bounded operating range is analytically proven and the proposed concept is verified using an OPAL-RT real-time digital simulation system.

Xiao et al., in [15], propose a zonal voltage control scheme based on multiple spatiotemporal characteristics for high penetration of PVs in active distribution networks (ADNs). The proposed method can achieve economic control and avoid overvoltage caused by errors in the forecast data of the PVs. For the spatial domain, zonal scheduling and zonal voltage control are carried out and the short-term scheduling and voltage controlling problem of the ADN is decomposed into several sub-problems. This simplifies the optimization and control which can reduce the computing time.

The article in [16] by Psillakis et al. develops a nonlinear coordinated excitation and static var compensator (SVC) control for regulating the output voltage and improving the transient stability of a synchronous generator infinite bus (SGIB) power system. A novel design is proposed that uses backstepping methodologies and feedback linearization techniques, suitably modified to take into account the delayed measurement feedback laws in order to implement both the excitation voltage and the SVC compensator input. A detailed and rigorous Lyapunov stability analysis reveals that if the time delays do not exceed some specific limits, then all closed-loop signals remain bounded and the frequency deviations are effectively regulated to approach zero.

In [17], Yao et al. address the integrated planning problem of a power network and the charging infrastructure of electric vehicles (EVs) for enhancing power system resilience under various extreme weather scenarios. The proposed coordinated planning framework is a robust two-stage/tri-level mixed-integer optimization model. The proposed robust joint planning model includes the construction plan in the first level, identifying the worst-case scenario in the second level, and optimizing the operation cost and load shedding in the final level. To solve this model, a duality-based column and constraint generation (D-CCG) algorithm is developed. Extended simulations validate the effectiveness of the approach.

In [18], Vlachakis et al. formulate a disturbance rejection problem of power-load step variations for an interconnected via weak tie-lines network system, formed of distinct control areas with identical dynamics. Load frequency control (LFC) is applied and analyzed by a centralized linear quadratic regulator (LQR) optimal design using a distributed scheme. Overall network stability is guaranteed via a stability test applied to a convex combination of Hurwitz matrices, the validity of which leads to stable network operation. The efficiency of the proposed distributed load frequency controller is illustrated via simulation studies.

Guo et al., in [19], propose a novel sliding mode control (SMC) as a control method which is insensitive to system uncertainties and disturbances to be applied in a hydraulic turbine governing system (HTGS). Dealing with certain fluctuations in speed and load around the rated condition, the proposed SMC is capable of driving the system to the desired state with smooth and light responses 
of the key state variables. Different operating scenarios are simulated and the results indicate smooth and robust responses.

A bi-level risk-limiting real-time unit commitment/real-time economic dispatch model with flexible ramping products (FRPs) is proposed in [20] by Lin et al. The model provides a new alternative to accommodate the high penetration of numerous intermittent renewable energy sources (IRESs). The modified IEEE 14-bus power system is employed to demonstrate the proposed method and the role of FRPs in enhancing the system.

In [21], Kontis et al., instead of analyzing the power system dynamics using accurate models, propose an identification toolbox for the derivation of measurement-based equivalent models and dynamic responses. The toolbox incorporates eight of the most widely used mode identification techniques. The capabilities of the toolbox are demonstrated using simulation responses, acquired from large-scale benchmark power systems, as well as using measurements recorded at a laboratory-scale active distribution network.

The concept of a compound attack detection mechanism, that links estimation-based and set-theoretic methods is addressed in [22] by Kontouras et al. The detection mechanism is developed as a security enhancing tool for the load-frequency control loop of a networked power system that consists of several interconnected control areas. Simulations indicate that an estimation-based detector is unable to discern an intermittent attack. The detector can be improved by exploiting the safe operation constraints imposed on the state variables of the system. Simulations indicate that the above detectors can operate jointly in terms of a hybrid scheme, which enhances their detection capabilities.

In the review presented in [23], Unruh et al. discussed several methods for the evaluation of different control approaches for grid-forming inverters. Grid-forming inverters are able to operate AC grids with or without rotating machines. Increasing shares of inverter-based electrical power generation, grid-forming inverters will become relevant for interconnected power systems. In contrast to conventional current-controlled inverters, grid-forming inverters do not immediately follow the grid voltage. They form voltage phasors that have an inertial behavior. In consequence, they can inherently deliver momentary reserve and increase power grid resilience.

Conflicts of Interest: The authors declare no conflict of interest.

\section{References}

1. Konstantopoulos, G.C.; Alexandridis, A.T.; Papageorgiou, P.C. Towards the Integration of Modern Power Systems into a Cyber-Physical Framework. Energies 2020, 13, 2169. [CrossRef]

2. Papageorgiou, P.C.; Alexandridis, A.T. Controlled Impedance-Admittance-Torque Nonlinear Modeling and Analysis of Modern Power Systems. Energies 2020, 13, 2461. [CrossRef]

3. Alexandridis, A.T. Studying State Convergence of Input-to-State Stable Systems with Applications to Power System Analysis. Energies 2020, 13, 92. [CrossRef]

4. Chen, L.; Nian, H.; Xu, Y. Impedance Aggregation Method of Multiple Wind Turbines and Accuracy Analysis. Energies 2019, 12, 2035. [CrossRef]

5. Chen, X.; Liu, Z. Impedance Modeling and Stability Analysis of the Converters in a Double-Fed Induction Generator (DFIG)-Based System. Energies 2019, 12, 2500. [CrossRef]

6. Luhtala, R.; Messo, T.; Roinila, T.; Alenius, H.; de Jong, E.; Burstein, A.; Fabian, A. Identification of Three-Phase Grid Impedance in the Presence of Parallel Converters. Energies 2019, 12, 2674. [CrossRef]

7. Petersen, L.; Iov, F.; Tarnowski, G.C.; Gevorgian, V.; Koralewicz, P.; Stroe, D.-I. Validating Performance Models for Hybrid Power Plant Control Assessment. Energies 2019, 12, 4330. [CrossRef]

8. Jauch, C.; Gloe, A. Simultaneous Inertia Contribution and Optimal Grid Utilization with Wind Turbines. Energies 2019, 12, 3013. [CrossRef]

9. Wang, B.; Cai, G.; Yang, D.; Wang, L.; Yu, Z. Investigation on Dynamic Response of Grid-Tied VSC During Electromechanical Oscillations of Power Systems. Energies 2020, 13, 94. [CrossRef] 
10. Peng, S.; Chen, H.; Lin, Y.; Shu, T.; Lin, X.; Tang, J.; Li, W.; Wu, W. Probabilistic Power Flow for Hybrid AC/DC Grids with Ninth-Order Polynomial Normal Transformation and Inherited Latin Hypercube Sampling. Energies 2019, 12, 3088. [CrossRef]

11. Li, X.; Li, Y.; Liu, L.; Wang, W.; Li, Y.; Cao, Y. Latin Hypercube Sampling Method for Location Selection of Multi-Infeed HVDC System Terminal. Energies 2020, 13, 1646. [CrossRef]

12. Wang, Y.; Zhou, Y.; Li, D.; Shao, D.; Cao, K.; Zhou, K.; Cai, D. The Influence of VSC-HVDC Reactive Power Control Mode on AC Power System Stability. Energies 2020, 13, 1677. [CrossRef]

13. von Meier, A.; Ratnam, E.L.; Brady, K.; Moffat, K.; Swartz, J. Phasor-Based Control for Scalable Integration of Variable Energy Resources. Energies 2020, 13, 190. [CrossRef]

14. Paspatis, A.G.; Konstantopoulos, G.C. Voltage Support under Grid Faults with Inherent Current Limitation for Three-Phase Droop-Controlled Inverters. Energies 2019, 12, 997. [CrossRef]

15. Xiao, C.; Sun, L.; Ding, M. Multiple Spatiotemporal Characteristics-Based Zonal Voltage Control for High Penetrated PVs in Active Distribution Networks. Energies 2020, 13, 249. [CrossRef]

16. Psillakis, H.E.; Alexandridis, A.T. Coordinated Excitation and Static Var Compensator Control with Delayed Feedback Measurements in SGIB Power Systems. Energies 2020, 13, 2181. [CrossRef]

17. Yao, F.; Wang, J.; Wen, F.; Tseng, C.-L.; Zhao, X.; Wang, Q. An Integrated Planning Strategy for a Power Network and the Charging Infrastructure of Electric Vehicles for Power System Resilience Enhancement. Energies 2019, 12, 3918. [CrossRef]

18. Vlahakis, E.; Dritsas, L.; Halikias, G. Distributed LQR Design for a Class of Large-Scale Multi-Area Power Systems. Energies 2019, 12, 2664. [CrossRef]

19. Guo, B.; Guo, J. Feedback Linearization and Reaching Law Based Sliding Mode Control Design for Nonlinear Hydraulic Turbine Governing System. Energies 2019, 12, 2273. [CrossRef]

20. Lin, H.; Wang, C.; Wen, F.; Tseng, C.-L.; Hu, J.; Ma, L.; Fan, M. Risk-Limiting Real-Time Economic Dispatch in a Power System with Flexibility Resources. Energies 2019, 12, 3133. [CrossRef]

21. Kontis, E.O.; Barzegkar-Ntovom, G.A.; Staios, K.A.; Papadopoulos, T.A.; Papagiannis, G.K. A Toolbox for Analyzing and Testing Mode Identification Techniques and Network Equivalent Models. Energies 2019, 12, 2606. [CrossRef]

22. Kontouras, E.; Tzes, A.; Dritsas, L. Hybrid Detection of Intermittent Cyber-Attacks in Networked Power Systems. Energies 2019, 12, 4625. [CrossRef]

23. Unruh, P.; Nuschke, M.; Strauß, P.; Welck, F. Overview on Grid-Forming Inverter Control Methods. Energies 2020, 13, 2589. [CrossRef] 